\title{
Contributions
}

Martin Gregor*

\section{To Invite or Not to Invite a Lobby, That Is the Question}

\begin{abstract}
We study a game in which a lobby with verifiable private evidence discloses her evidence to a policy-maker if and only if she agrees to a transfer that is proposed by the policy-maker. This setting is motivated by the literature of pay-and-lobby politics, which finds that politicians schedule informative meetings with lobbyists on the basis of their campaign contributions. We admit both positive transfers (fees) and negative transfers (compensations), which implies not only that the policy-maker can commit to not listen but also that the lobby can commit to not talk. In a binary action space, we solve the game for all timings, prior beliefs, information structures, and valuation parameters. We identify the settings in which the policy-maker strategically discourages the lobby's participation by announcing an unacceptable transfer. Whether 'burying one's head in the sand' increases or decreases welfare depends on the degree of the policy-maker's benevolence.
\end{abstract}

Keywords: disclosure, hard evidence, access fee, lobbying, attention

DOI 10.1515/bejte-2014-0022

\section{Introduction}

In games with strategic information transmission of verifiable evidence, a standard assumption is that all of a sender's messages are observed by a receiver (Milgrom 1981; Milgrom and Roberts 1986; Seidmann and Winter 1997; Dewatripont and Tirole 2005). In many real-world situations, however, attention is limited and must be allocated. The receiver does not observe all messages and

\footnotetext{
*Corresponding author: Martin Gregor, Faculty of Social Sciences, Institute of Economic Studies, Charles University in Prague, IES FSV UK, Opletalova 26 11000, Prague CZ, E-mail: gregor@fsv.cuni.cz http://orcid.org/0000-0002-7657-724X
} 
follows a pre-determined rule that selects the set of observable messages. A particularly interesting class of selection rules is that of rules that condition the observation of a message on a transfer between the sender and the receiver.

In this article, we focus on the disclosure of verifiable evidence between a single informed lobby and an uninformed policy-maker. In this particular context, observation of the evidence may require that the policy-maker and the lobby agree on a meeting. A simple version of the rule for a meeting is when the policy-maker announces an access fee that a lobby must pay if she wants to meet the policy-maker (Austen-Smith 1995, 1998; Ball 1995; Lohmann 1995; Cotton 2012). The policy-maker's motivation to use such a rule is to exploit the lobby's gains that emerge when the policy-maker learns new information and makes a decision in favor of the communicating lobby.

The access fee mechanism may explain the close links between campaign spending and lobbying. Indeed, a large body of literature on 'pay-and-lobby' or 'pay-to-play' politics argues that politicians allocate a significant part of their attention to listening to hard evidence delivered by special interest groups on the basis of their campaign contributions while striving to maintain a reputation of sticking to non-contractible actions (Baron 1989; Snyder 1990; Wright 1996; Cotton 2012). Empirically, the idea that 'money buys access' is supported by evidence of the link between campaign contributions and lobbying outlays on the level of both donors and recipients (Ansolabehere, Snyder, and Tripathi 2002; Esterling 2007).

Nevertheless, the access fee mechanism empowers the policy-maker with the ability to extract extra surplus, while the lobby has no countervailing power. To make things symmetric, we consider a broader situation where both players can set a condition for a meeting; to make the condition credible, the policymaker has a commitment to not listen, and the lobby disposes with a commitment to not talk. The double commitment is modeled simply; the domain of the transfer proposed by the policy-maker includes both positive transfers (fees) as well as negative transfers (compensations).

Our setting is thus general in a sense that it permits both fees and compensations for access. To achieve generality, we consider both relevant timings: the payment of the fee may precede or follow the realization of the private signal. All strategic variables have binary support, which allows us to solve the game conveniently in a complete parametrical space and for all information structures. As a robustness check, we solve the game under alternative assumptions that only fees or only compensations are feasible, and we also consider plain commitments that are not conditional on the transfer.

We have two main objectives. First, we examine whether the policy-maker proposes an acceptable or unacceptable (prohibitive) transfer. In the latter case, 
the lobby disagrees with the proposal, and the information is not transmitted. The existence of prohibitive transfers may shed light on why many stakeholders abstain from lobbying and do not provide verifiable evidence. ${ }^{1}$ From the perspective of the classic disclosure game (Milgrom 1981; Milgrom and Roberts 1986), the absence of communication indicates pooling efforts of low types of lobbies. Our model with a double commitment explains the absence of communication not as a lack of favorable evidence but as the presence of a very attractive status quo outside option of the lobby.

To understand the policy-maker's incentive to announce a prohibitive transfer, consider the following scenario: Having only prior beliefs, the policymaker selects an action that is optimal for the lobby independently of the state of the world. The lobby is invited before she learns private information. Under such circumstances, the lobby participates only if she is compensated for the 'disclosure experiment' by means of an access compensation. If such a compensation is prohibitively costly for the policy-maker, then the policy-maker announces a lower compensation, which the lobby does not accept. We find that this scenario indeed describes the only situation in which the information is sacrificed.

This result is an initial step in understanding the policy-maker's optimal transfers in more complex situations that reach beyond the scope of our paper. Most importantly, with multiple lobbies, the policy-maker may discourage the participation of the lobby for a different reason; namely, the participation of one lobby decreases the level of transfer in communication with another lobby. In other words, some lobbies are discouraged to participate because the extra verifiable evidence of these uninvited lobbies would imply a prohibitive loss in the interactions with the invited lobbies. These external effects are absent in our setting with a single lobby.

Our second main objective is to examine the welfare consequences of the lack of communication. We use two welfare measures that differ in the interpretation of the policy-maker's objective, and we reach two clear results: If the policy-maker is non-benevolent, then the lack of communication implies a social gain. Intuitively, the commitment power of the lobby induces the policy-maker to internalize externalities of his non-contractible actions. In contrast, if the policy-maker has a mixed objective that involves benevolence and private benefits, as in common-agency models (c.f., Grossman and Helpman 2001), then externalities are absent, and the access restriction implies a social loss.

1 Richter, Samphantharak, and Timmons (2009) show that only a small fraction of firms actually lobby and that lobbying expenditures follow a skewed, power-law distribution. Kerr, Lincoln, and Mishra (2011) find that lobbying status is persistent over time. 
The welfare assessment of incomplete participation is thus determined by the policy-maker's objective.

The paper is most closely related to the analysis of endogenous access fees in the presence of verifiable evidence, where verifiability implies that the credibility of the lobby's message is not related to the lobby's bias (Austen-Smith 1998; Cotton 2009, 2012). Austen-Smith (1998) considers two lobbies and endogenous noise structures. Cotton (2012) assumes two lobbies as well, but the information structure is exogenous and noiseless. This paper considers only a single lobby but is more comprehensive in the analysis of all scenarios and in the discussion of the welfare effect relative to various benchmarks. Moreover, our setting allows us to directly compare various results based on different timings.

This paper can be also seen as an extension of the classic disclosure game (Milgrom 1981; Milgrom and Roberts 1986; Shin 1994; Seidmann and Winter 1997; Dziuda 2011; Bhattacharya and Mukherjee 2013) by conditional commitments to not listen and not talk. Our setup demonstrates how to calculate the equilibrium participation by means of bilateral surpluses, which may serve as a toolkit for modeling disclosure with conditional commitments under more complex settings with more lobbies and a richer state space. Because the model also covers ex-ante access whereby the participation decision is a decision to run or not run a public experiment, the model can also be combined with lobbying models that include a search for hard evidence (Bennedsen and Feldmann 2006; Dahm and Porteiro 2008).

The paper proceeds as follows: Section 2 builds the setup and explains the role of the double commitment. Section 3 identifies the equilibrium and provides a comparative statics analysis. It also solves the equilibria in alternative regimes. Section 4 makes a welfare assessment of the access restriction and the consequent information loss. Section 5 discusses the players' preferences regarding noise and the equilibrium when the refinements are relaxed. Section 6 concludes.

\section{Setup}

\subsection{Assumptions}

\subsubsection{State of Nature and signal}

Consider a binary state of Nature, $\theta \in\{0,1\}$. The common prior belief is $\pi_{\varnothing}:=\operatorname{Pr}(\theta=1) \in(0,1)$. There is a binary signal $s \in\{0,1\}$ characterized 
by a pair of Type-I and Type-II errors, $\alpha \in[0,1)$ and $\beta \in[0,1)$, where $\alpha:=\operatorname{Pr}(s=0 \mid \theta=1)$ and $\beta:=\operatorname{Pr}(s=1 \mid \theta=0)$. The posterior for a signal $s$ is $\pi_{s}:=\operatorname{Pr}(\theta=1 \mid s)$. Theunconditional probability of high signal realizations is $f\left(\alpha, \beta, \pi_{\varnothing}\right):=\operatorname{Pr}(s=1)=(1-\alpha) \pi_{\varnothing}+\beta\left(1-\pi_{\varnothing}\right)$. Without a loss of generality, suppose a positive correlation between the signals and the states of the world. Formally, the posteriors satisfy $\pi_{1} \geq \pi_{\emptyset} \geq \pi_{0}$, which is equivalent to

$$
\alpha+\beta \leq 1 \text {. }
$$

\subsubsection{Players}

There is a lobby (she) and a policy-maker (he). The lobby privately observes $s$. The signal contains hard (verifiable) evidence that cannot be fabricated but that may not be disclosed. We consider the lobby to be a low type if $s=0$ and a high type if $s=1$.

\subsubsection{Strategies}

The policy-maker proposes a minimum transfer from the lobby to the policymaker, $c_{P} \in \mathbb{R}$. (Notice that the proposed transfer may be negative.) The policymaker also selects a binary action (policy) $a \in\{0,1\}$. The lobby proposes a maximal transfer from the lobby to the policy-maker, $c_{L} \in \mathbb{R}$. If the two proposals are consistent, $c_{L} \geq c_{P}$, then the lobby subsequently makes a decision regarding disclosure or non-disclosure, $d \in\{0,1\}$. Neither player's actions are contractible.

\subsubsection{Objectives}

The lobby always prefers high action $a=1$. Her state-independent value of high action relative to low action is normalized to unity. The policy-maker prefers high action if $\theta=1$, and he prefers low action if $\theta=0$. Namely, we let $L:\{0,1\} \times\{0,1\} \rightarrow\{0, V\}$ be the policy-maker's loss when adopting action $a$ in state $\theta, L(a, \theta):=(\theta-a)^{2} V$, where $V>0$. The loss function is zero if $a=\theta$ and is equal to $V$ if $a \neq \theta$; hence, the loss function is symmetric in both states of Nature. The expected loss $E:\{0,1\} \times[0,1] \rightarrow[0, V]$ is the expected value of the policy-maker's loss when adopting action $a$ under beliefs $\pi$, $E(a, \pi):=(1-\pi) L(a, 0)+\pi L(a, 1)$. If the expected losses are equal, the policymaker is assumed to select the low action. 


\subsubsection{Timing}

In Stage 1, the policy-maker makes a proposal, $c_{P} \in \mathbb{R}$. In Stage 2, the lobby makes a counterproposal, $c_{L} \in \mathbb{R}$. The effective transfer is $c=c_{L} \cdot \mathbf{1}\left\{c_{L} \geq c_{P}\right\}$. By strict dominance, the action space of the lobby can be simplified into a binary decision $^{2}$ regarding participation, $e \in\{0,1\}$, where the effective payment is $c=e \cdot c_{P}$. In Stage 3 , if the lobby participates, she makes a decision regarding the disclosure of the evidence, $d \in\{0,1\}$. In Stage 4 , the effective transfer is realized and the policy-maker selects action $a \in\{0,1\}$.

Two timings of the signal realization are relevant: (i) Under ex-ante access, the signal is realized between Stage 2 and Stage 3. The entry decision is made by the lobby without private information. (ii) Under interim access, the signal is realized between Stage 1 and Stage 2. For this timing, the entry decisions are made by the two types of lobbies just after the signal is realized.

\subsubsection{Lobby’s strategies}

For any timing, disclosure is decided by the type of the lobby. We distinguish between the low type of the lobby's disclosure $d_{0} \in\{0,1\}$ and the high type of the lobby's disclosure $d_{1} \in\{0,1\}$. Under ex-ante access, entry is decided by the lobby, $e \in\{0,1\}$. Under interim access, entry is decided by the type of the lobby, and we distinguish between $e_{0} \in\{0,1\}$ and $e_{1} \in\{0,1\}$.

\subsubsection{Indifferences}

We treat all indifferences in favor of participation: (i) If the lobby is indifferent over entry, she enters. (ii) If the policy-maker is indifferent over a set $\left\{c_{P}^{1}, c_{P}^{2}, \ldots\right\}$, he sets $c_{P}=\max \left\{c_{P}^{1}, c_{P}^{2}, \ldots\right\}$. This has the advantage that the lack of participation in the equilibrium indicates the positive benefits of non-participation.

\subsubsection{Signals}

Given symmetry in the loss function, the policy-maker's preferred action under belief $\pi$ is determined easily. From minimization of the expected loss, we

2 If $c_{L} \geq c_{P}$, the condition to proceed to Stage 3 is met. The lowest $c_{L}$ consistent with the condition gives $c=c_{L}=c_{P}$. 
determine that the low action is selected under belief $\pi$ if and only if $E(0, \pi) \leq E(1, \pi)$, which is equivalent with $\pi \leq \frac{1}{2}$. We use this inequality to classify signals by a standard informativeness criterion. A signal is uninformative if the policy-maker selects identical actions after observing any signal realization. Otherwise, the signal is informative. The exact shape of the criterion depends on the prior:

- If $\pi_{\varnothing}>\frac{1}{2}$, then $\pi_{1}>\frac{1}{2}$. Informativeness depends on $\pi_{0} \leq \frac{1}{2}$ only. ${ }^{3}$ This is captured by the indicator $I\left(\alpha, \beta, \pi_{\phi}\right):=\mathbf{1}\left\{\beta \leq 1-\alpha \frac{\pi_{\phi}}{1-\pi_{\phi}}\right\}$.

- If $\pi_{\varnothing} \leq \frac{1}{2}$, then $\pi_{0} \leq \frac{1}{2}$. Informativeness depends on $\pi_{1}>\frac{1}{2}$ only. We introduce the indicator $I^{\prime}\left(\alpha, \beta, \pi_{\varnothing}\right):=\mathbf{1}\left\{\beta<(1-\alpha) \frac{\pi_{\phi}}{1-\pi_{\varnothing}}\right\}$.

\subsection{Double commitment and a bilateral transaction}

If the lobby participates, a disclosure subgame involves the attention of both the policy-maker and the lobby. Thus, our game can be seen as a bilateral transaction, where both parties bring attention to the table and one party also brings money to the table. A normal version of the transaction is that the policy-maker trades his attention (willingness to listen), while the lobby offers both her attention (willingness to talk) and a payment. Nevertheless, our mechanism also permits transactions in which the policy-maker offers the payment. Thus, our paper is a generalization in a sense that it permits bilateral transactions of any kind, and this generalization deserves extra discussion.

Is our general mechanism with both positive transfers (access fees) and negative transfers (access compensations) realistic? In the following analysis, we derive the necessary conditions for the implementation of both types of transfers. Formally, we analyze which restrictions upon the player's actions in our game are necessary if we compare our game with a game in which the timing is identical but no restrictions on the action space exist in Stage 3. In the non-restricted game, Stage 3 can be modeled as a simultaneous game in which the lobby's message space is $\{\emptyset, 0,1\}$ and the policy-maker's attention space is \{listen, not listen $\}$.

The first necessary restriction for the implementation of our game is the existence of the policy-maker's conditional commitment to not listen. (Conditionality means that an action is conditional upon the transfer

3 Recall that $\pi_{0}$ is defined everywhere by the Bayes rule because we have disregarded the corner signal $(\alpha, \beta)=(0,1)$. 
realization.) In the absence of this commitment, the policy-maker's weakly dominant action in Stage 3 is to listen. This limits the policy-maker's opportunity to extract access fees. The second necessary restriction is the lobby's conditional commitment to not talk. In the absence of this commitment, if the policy-maker is expected to listen, then under skeptical beliefs, $m=\emptyset$ is interpreted as a low type, and hence, the lobby reveals her information. This limits the lobby's opportunity to extract access compensations, and the game boils down to an access fee game in which only the scenario with access fees is feasible.

Our mechanism is therefore implemented only if the policy-maker can conditionally commit to not listen and the lobby can conditionally commit to not communicate. For each player, the condition of the commitment is that a realized transfer is consistent with the player's proposal. The existence of the double conditional commitment gives each trading party a disagreement point that is independent of the action of the other party and of the level of the proposed fee. Thus, we can indeed interpret the game as a bilateral transaction.

The two commitments are not active at the same time, however. If the proposed transfer is positive and the policy-maker is the beneficiary, only the policy-maker's commitment is active. If the proposed transfer is negative and the lobby is the beneficiary, only the lobby's commitment is active.

How should one interpret the double commitment in the context of lobbying? The policy-maker's commitment device is often attributed to the existence of tight schedules. In the absence of free time slots, the policymaker cannot devote the minimal required attention to the extra policy agenda and must rely on his (prior or skeptical) belief. The lobby's commitment device might originate in circumstances when an extra action is necessary before communication. For example, in many agendas, hiring a connected agent in advance is a precondition of successful lobbying (Bertrand, Bombardini, and Trebbi 2014). Alternatively, the lobby's private signal may not be verifiable, and the lobby needs to generate the verifiable signal in advance to be able to communicate the message.

Finally, the transfers must be carefully interpreted. While fees are normally interpreted to be campaign contributions, compensations may be in the form of base earmarks or subsidies that the policy-maker can credibly provide. The credibility of the negative transfer may rise in particular when the policy-maker provides informational benefits (e.g. departmental reports, regulatory prospects, or any other valuable data about a topic separate from the analysis). In such a case, the policy-maker offers a meeting to discuss this information with the lobby, and during such a meeting, the lobby cannot avoid being subject to the questions that imply the disclosure of her type. 


\section{The relevant equilibrium}

\subsection{Two skeptic refinements}

We will show that both timings can be solved in an identical way. For this purpose, we apply two standard refinements. (The equilibria that do not comply with these standard refinements are analyzed in the extensions.) Both refinements intuitively mean that the policy-maker has skeptical beliefs. As a skeptic, the policy-maker interprets non-participation or non-disclosure to be a signal of the low type of the lobby, as long as such an equilibrium exists.

First, in the disclosure stage, we restrict ourselves to profiles for which both types of lobbies are willing to disclose their evidence. To determine these profiles safely, we set the out-of-equilibrium belief for non-disclosure $d=0$ as the minimal posterior $\pi_{0}$. Intuitively, non-disclosure is interpreted to characterize a low type of the lobby. Under these beliefs, the low type of the lobby is indifferent between disclosure and non-disclosure because both induce an identical posterior from the policy-maker. This assumption complies with the intuitive criterion of Cho and Kreps (1987).

Assumption 1 (Disclosure) We select an equilibrium with full disclosure, $\left(d_{0}, d_{1}\right)=(1,1)$, and set the out-of-equilibrium posterior $\operatorname{Pr}(\theta=1 \mid d=0)=\pi_{0}$, if such an equilibrium exists.

The next refinement applies to interim access only, where signaling subgames that begin with a private observation may involve two pure-strategy equilibria. In an equilibrium, either both types of lobbies are pooled, $\left(e_{0}, e_{1}\right)=(0,0)$, or both types are separated, $\left(e_{0}, e_{1}\right)=(0,1)$. In the spirit of the unraveling argument by Milgrom (1981), we focus on the separating equilibrium only, if separation exists.

Assumption 2 (Separation) Under interim access, we select a separating equilibrium, $\left(e_{0}, e_{1}\right)=(0,1)$, if such an equilibrium exists.

\subsection{Bargaining perspective}

A helpful perspective upon the game is a transaction (bargaining) perspective where both the policy-maker and the lobby must agree to the transfer so that the evidence is disclosed. The application of the transaction perspective depends on timing, because timing determines the identity of the bargaining partner of the 
policy-maker. For ex ante access, the lobby is the bargaining partner. For interim access, only the high type of the lobby is the bargaining partner. ${ }^{4}$ Given the change in the identity, timing affects the outside option of the partner, the partial gains of the partner, the partial gains of the policy-maker, and the bargaining surplus.

The bargaining protocol is a single take-it-or-leave-it offer: In Stage 1, the policy-maker makes an offer. In Stage 2, the partner agrees or disagrees. The equilibrium under this protocol is easily found as follows:

1. Calculate the positive or negative partial gains of the agreement (a.k.a. values of access) for each bargaining party. The sum of the partial gains defines the bargaining surplus.

2. If the surplus is non-negative, the policy-maker offers a transfer that exactly meets the partner's participation condition. We say that the policy-maker invites his partner. Therefore, the policy-maker extracts all partial gains of the partner and seizes full surplus. The partner is left with her outside option.

3. If the surplus is negative, the policy-maker offers a prohibitively high transfer. We say that the policy-maker does not invite his partner. The partner disagrees.

\subsection{Bargaining surplus}

Consider first the policy-maker. His outside option is independent of the timing of the signal realization. Namely, his outside option is described by her prior beliefs and the action corresponding to her prior beliefs, to be denoted $a_{\varnothing}$. Clearly, we have $a_{\varnothing}=\mathbf{1}\left\{\pi_{\varnothing}>\frac{1}{2}\right\}$. At the outside option, his expected loss is $E_{\varnothing}\left(\pi_{\varnothing}\right):=E\left(a_{\varnothing} ; \pi_{\varnothing}\right)=\min \left\{\pi_{\varnothing}, 1-\pi_{\varnothing}\right\} V$.

If the partner agrees, the policy-maker learns new information, updates his beliefs, and may change his action. For an uninformative signal, there is no effect on the policy-maker's action. Therefore, the policy-maker's partial gain is zero. For an informative signal, the expected loss drops from $E_{\varnothing}\left(\pi_{\varnothing}\right)$ to $E_{e}\left(\alpha, \beta, \pi_{\varnothing}\right):=\left[\alpha \pi_{\varnothing}+\beta\left(1-\pi_{\varnothing}\right)\right] V$.

To obtain the remaining bargaining variables, we have to distinguish between the timings of the signal realization.

4 With skeptic beliefs, the policy-maker needs only one type of the lobby to participate. The participation condition of the low type of the lobby is more restrictive; hence, the policy-maker sets a transfer that meets participation condition of the high type of the lobby. 


\subsubsection{Ex ante access}

The lobby is the bargaining partner. The outside option of the lobby is the status-quo action $a_{\varnothing}$. For an uninformative signal, there is no effect on the action; hence, the lobby's partial gain is zero. For an informative signal, the probability of favorable action $a=1$ changes to $f\left(\alpha, \beta, \pi_{\varnothing}\right)$, and the gain is $f\left(\alpha, \beta, \pi_{\varnothing}\right)-\mathbf{1}\left\{\pi_{\varnothing}>\frac{1}{2}\right\}>0$. The bargaining surplus for ex ante access, denoted as $S\left(\alpha, \beta, \pi_{\varnothing}\right)$, is a sum of both partial gains:

$$
S\left(\alpha, \beta, \pi_{\varnothing}\right):=\left\{\begin{array}{cc}
I\left(\alpha, \beta, \pi_{\varnothing}\right)\left[E_{\varnothing}\left(\pi_{\varnothing}\right)-E_{e}\left(\alpha, \beta, \pi_{\varnothing}\right)+f\left(\alpha, \beta, \pi_{\varnothing}\right)-1\right] \gtreqless 0 & \text { if } \pi_{\varnothing}>\frac{1}{2}, \\
I^{\prime}\left(\alpha, \beta, \pi_{\varnothing}\right)\left[E_{\varnothing}\left(\pi_{\varnothing}\right)-E_{e}\left(\alpha, \beta, \pi_{\varnothing}\right)+f\left(\alpha, \beta, \pi_{\varnothing}\right)\right] \geq 0 & \text { if } \pi_{\varnothing} \leq \frac{1}{2} .
\end{array}\right.
$$

\subsubsection{Interim access}

The high type of the lobby is the bargaining partner. By Assumption 2, the outside option of the high type of the lobby is no longer the status-quo option but rather is unfavorable action $a=0$. The gain of participation is thus unity. Since the high type occurs only $f$-times, her gain must be normalized by $f\left(\alpha, \beta, \pi_{\varnothing}\right)$. The bargaining surplus for interim access where only the high type pays for access, denoted as $H\left(\alpha, \beta, \pi_{\varnothing}\right)$, is:

$$
H\left(\alpha, \beta, \pi_{\varnothing}\right):= \begin{cases}I\left(\alpha, \beta, \pi_{\varnothing}\right)\left[E_{\varnothing}\left(\pi_{\varnothing}\right)-E_{e}\left(\alpha, \beta, \pi_{\varnothing}\right)+f\left(\alpha, \beta, \pi_{\phi}\right)\right] \geq 0 & \text { if } \pi_{\varnothing}>\frac{1}{2} \\ I^{\prime}\left(\alpha, \beta, \pi_{\varnothing}\right)\left[E_{\varnothing}\left(\pi_{\varnothing}\right)-E_{e}\left(\alpha, \beta, \pi_{\varnothing}\right)+f\left(\alpha, \beta, \pi_{\varnothing}\right)\right] \leq 0 & \text { if } \pi_{\varnothing}<\frac{1}{2}\end{cases}
$$

Under interim access, agreement is more likely, because the surplus cannot be smaller than under ex ante access,

$$
H\left(\alpha, \beta, \pi_{\varnothing}\right)=S\left(\alpha, \beta, \pi_{\varnothing}\right)+\mathbf{1}\left\{\pi_{\varnothing}>\frac{1}{2}\right\} \cdot I\left(\alpha, \beta, \pi_{\varnothing}\right) \geq S\left(\alpha, \beta, \pi_{\varnothing}\right) .
$$

\subsection{Lack of communication}

We use the surpluses to identify the necessary conditions for a negative value of the surplus. Under our treatment of indifferences that favor participation, a negative surplus is equivalent to setting a prohibitive transfer. 
Proposition 1 (Prohibitive transfer) The policy-maker sets a prohibitive transfer only if access is ex ante, the status-quo action is the high action, $a_{\varnothing}=1$, and the signal is informative, $I\left(\alpha, \beta, \pi_{\varnothing}\right)=1$.

Proof. Using the bargaining perspective, a sufficient and necessary condition for a prohibitive transfer is negative surplus. To identify the negative surplus, we prove inequalities in eqs [2] and [3]. It is sufficient to recall that $f\left(\alpha, \beta, \pi_{\varnothing}\right) \geq 0$ and $E_{\varnothing}\left(\pi_{\varnothing}\right) \geq E_{e}\left(\alpha, \beta, \pi_{\varnothing}\right)$. The surplus can be negative only in the first case of eq. [2], where access is ex ante, the status quo is favorable for the lobby, and the signal is informative.

\subsection{Noise and prohibitive transfer}

When the bargaining surplus is negative, the policy-maker cannot compensate the lobby for her participation without making himself worse off. The source of the negative surplus lies in the attractiveness of the lobby's outside option, which makes the agreement to disclose the evidence prohibitively costly.

In this section, we focus in detail on the existence of the prohibitive transfer in the parametrical space $\left(\alpha, \beta, \pi_{\varnothing}, V\right)$. By Proposition 1 , it is sufficient to consider only the timing under ex ante access and the case of $\pi_{\varnothing}>\frac{1}{2}$, which implies favorable status-quo action $a_{\varnothing}=1$.

In the first step, we find that for any $V>0$, a set of informative signals exists such that the access is strategically restricted. Such signals are found in the neighborhood of the informative signal with the maximal $\alpha$-error and minimal $\beta$-error, $(\alpha, \beta)=\left(\frac{1-\pi_{\emptyset}}{\pi_{\emptyset}}, 0\right)$. This result occurs because the corresponding surplus with this signal is negative,

$$
S\left(\frac{1-\pi_{\phi}}{\pi_{\varnothing}}, 0, \pi_{\varnothing}\right)=-2\left(1-\pi_{\varnothing}\right)<0 .
$$

To understand the role of noise in more detail, we examine the iso-surplus curves. The iso-surplus curves are linear in the signal space $(\alpha, \beta)$, because the partial derivatives are independent of $(\alpha, \beta)$,

$$
\begin{gathered}
\frac{\partial S\left(\alpha, \beta, \pi_{\varnothing}\right)}{\partial \alpha}=-\pi_{\varnothing}(V+1)<0, \\
\frac{\partial S\left(\alpha, \beta, \pi_{\varnothing}\right)}{\partial \beta}=-\left(1-\pi_{\varnothing}\right)(V-1) \gtreqless 0 .
\end{gathered}
$$


The key curve is the zero-surplus curve, $S\left(\alpha, \beta, \pi_{\varnothing}\right)=0$. By simple algebra, the curve is defined by

$$
\beta=1-\alpha \frac{\pi_{\varnothing}}{1-\pi_{\varnothing}} \frac{V+1}{V-1} .
$$

The zero-surplus curve always originates from the NW corner, because $S\left(0,1, \pi_{\varnothing}\right)=0$. The shape of the curve depends on $V \gtreqless 1$ :

- The policy-maker's valuation exceeds the lobby's valuation $(V>1)$ : From eqs [6] and [7], the iso-surplus curves move from NW to SE. Informative signals with low noise have a positive surplus, and informative signals with large noise have a negative surplus.

- Both valuations are identical $(V=1)$ : The iso-surplus curves move from $\mathrm{N}$ to S. Hence, informative signals with $\alpha=0$ have exactly a zero surplus. Any other informative signal features a negative surplus.

- The lobby's valuation exceeds the policy-maker's valuation $(V<1)$ : The isosurplus curves move from NE to SW. All informative signals have a negative surplus.

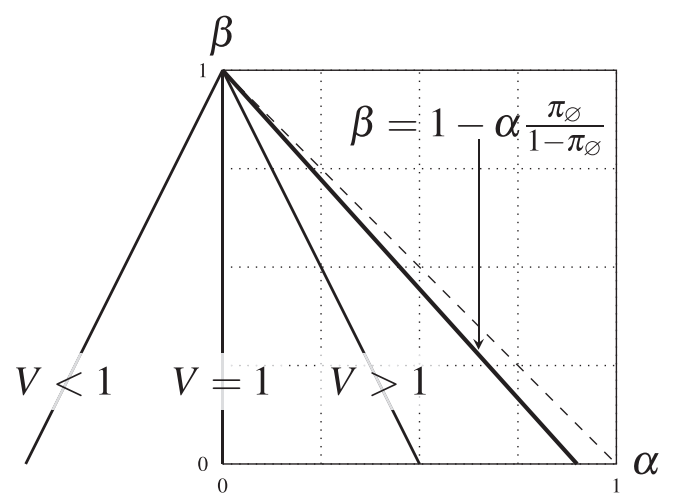

Figure 1: The shape of the zero-surplus curve for $\pi_{\emptyset} \geq \frac{1}{2}$

Figure 1 illustrates. In the signal space, we thus recognize three types of signals:

- Small noise: The signal is informative, and the surplus is non-negative. In the equilibrium, the lobby participates. For $V<1$, the set is empty.

- Moderate noise: The signal is informative, but the surplus is negative. In the equilibrium, participation is prohibitively costly for the lobby. The set is non-empty. 
- Large noise: The signal is uninformative, and the surplus is zero. In the equilibrium, the status quo is maintained. The set is non-empty.

The surplus is non-monotonic at a structural switch from an informative to an uninformative signal. Namely, at $\beta=1-\alpha \frac{\pi_{\phi}}{1-\pi_{0}}$, the negative surplus changes into a zero surplus. This non-monotonicity associated with a structural switch is strategically relevant in the setting in which signals are endogenous to the players (see Section 5).

\subsection{Alternative regimes}

Our model considers commitments that are conditional on the realization of the transfer; the policy-maker is committed to not listen, and the lobby is committed to not communicate if the transfer is not realized. What if a certain commitment is missing? This case is analyzed in Table 1. The table also considers plain commitments that are not conditional on the transfer. Table 1 solves the equilibria for alternative regimes, depending on the structure of commitments (none, unilateral, bilateral) and the type of commitments (plain, conditional).

Table 1: The equilibria in alternative regimes

\begin{tabular}{|c|c|c|c|}
\hline Regime $\backslash$ The lobby's gains; Surplus & $\begin{array}{l}\text { Positive, } \\
\text { positive }\end{array}$ & $\begin{array}{l}\text { Negative, } \\
\text { positive }\end{array}$ & $\begin{array}{l}\text { Negative, } \\
\text { negative }\end{array}$ \\
\hline No commitment (Benchmark 1) & Disclosure & Disclosure & Disclosure \\
\hline Plain commitment to not listen & Disclosure & Disclosure & Disclosure \\
\hline Plain commitment to not talk & Disclosure & Non-disclosure & Non-disclosure \\
\hline Two plain commitments (Benchmark 2) & Disclosure & Non-disclosure & Non-disclosure \\
\hline Conditional commitment to not listen & Disclosure, $c>0$ & Disclosure & Disclosure \\
\hline Conditional commitment to not talk & Disclosure & Disclosure, $c<0$ & Non-disclosure \\
\hline $\begin{array}{l}\text { Two conditional commitments } \\
\text { (our game) }\end{array}$ & Disclosure, $c>0$ & Disclosure, $c<0$ & Non-disclosure \\
\hline
\end{tabular}

Note: The effective transfer is zero $(c=0)$ unless written otherwise.

We have three configurations of preferences that depend on the sign of the lobby's gains and the sign of the surplus. If both commitments are present, then in each configuration, exactly one of the two commitments is active and one is passive. Making the passive commitment absent has no effect on the equilibrium; only the absence of the active commitment matters. 
Interestingly, the type of the commitment fully characterizes the effect of its absence. By making the commitment to not listen passive, the effect is redistributive but not informative. In contrast, by making the commitment to not talk passive, the effect is informative but not redistributive. A consequence of this property is that a loss of the commitment to not listen has no effect on the equilibrium if the commitments are plain; such a loss may have only a redistributive effect, but redistribution is by definition absent for plain commitments.

Additionally, because the sum of the player's utilities is measured by the amount of information, only the existence of the commitment to not talk may affect the sum. (This observation is used in the utilitarian welfare analysis in the following Section 4.) From that perspective, only the lobby's commitment is essential for understanding the strategic non-participation.

Notice that the regimes with a unilateral conditional commitment can be achieved by augmenting our game through restricting the action space of the proposed transfers. The regime with a unilateral commitment to not listen is equivalent to the assumption that the proposed transfer is non-negative, $c_{P} \geq 0$ (only access fees), and the regime with a unilateral commitment to not talk is equivalent to the assumption that the proposed transfer is non-positive, $c_{P} \leq 0$ (only access compensations).

Finally, we can discuss the effects of the introduction of transfers; this can be interpreted such that plain commitments (Benchmark 2) turn into conditional commitments. As Table 1 reveals, the introduction of transfers implies that the positive surplus, which was previously not generated (because of the impossibility of compensating the lobby), is now seized by the policy-maker.

\section{Welfare}

We apply two alternative normative analyses. In the first analysis, we treat our model in a standard utilitarian framework. The players' utilities are independent (a non-benevolent policy-maker), and the utilitarian welfare is maximized if the sum of the policy-maker's and the lobby's utilities is maximized. In this setting, the bilateral surplus is a welfare measure. In the second setting, we adopt a specification from common agency modeling (Grossman and Helpman 2001) in which the policy-maker's objective contains welfare generated out of the actions and private gains from revenue (a benevolent policy-maker). Here, the bilateral surplus is not a welfare measure.

We will observe two types of potential welfare distortions: (i) The policymaker may neglect the negative externality of her action $a$ on the lobby. We will 
show that the negative externality is relevant only if the policy-maker is nonbenevolent. (ii) There is an information loss associated with strategic access restriction resulting in a status-quo bias. We will show that the information loss is relevant only if the policy-maker is benevolent.

Given that the two settings generate two opposite welfare distortions, we will also find a completely opposite prediction for the effect of the prohibitive transfer on welfare. As a benchmark, consider a setting where no commitments are available (Benchmark 1 in Table 1). Our main result is that when the policymaker is non-benevolent, our mechanism (double commitment) enhances welfare relative to the benchmark, whereas when the policy-maker is benevolent, our mechanism deteriorates welfare relative to the benchmark.

\subsection{Non-benevolent policy-maker}

We may represent the lobby's objective as a loss function $l:\{0,1\} \rightarrow\{0,1\}$, where $l(a)=1-a$. The utilitarian objective minimizes the sum of the policymaker's expected loss and the lobby's expected loss. We first derive the firstbest actions, $\left(a_{0}^{*}, a_{1}^{*}\right)$. For a signal $s$ and corresponding beliefs $\pi_{s}$, the first-best action is

$$
a_{s}^{*}=\arg _{a \in\{0,1\}} \min \left\{E\left(a, \pi_{s}\right)+l(a)\right\}=\arg _{a \in\{0,1\}} \min \left\{\pi_{s} V+1 ;\left(1-\pi_{s}\right) V\right\} .
$$

This expression can be reduced to

$$
a_{s}^{*}= \begin{cases}0 & \text { if } \pi_{s} \leq \frac{V-1}{2 V}, \\ 1 \quad \text { if } \pi_{s}>\frac{V-1}{2 V} .\end{cases}
$$

Notice that $\frac{V-1}{2 V}<\frac{1}{2}$. Next, since posteriors are non-decreasing in the lobby's type, $\pi_{1}^{*} \geq \pi_{0}^{*}$, the optimal actions are also non-decreasing, $a_{1}^{*} \geq a_{0}^{*}$. Recall that the equilibrium actions are also non-decreasing in the lobby's type, $a_{1} \geq a_{0}$.

We may expect two sources of welfare distortion:

- In her ex post decision, the policy-maker neglects the negative externality of a low action on the lobby. Hence, the policy-maker is biased toward low action for any signal realization. Nevertheless, the bias does not need to be strong enough to make a difference between the equilibrium action $a_{s}$ and the first-best action $a_{s}^{*}$.

- In her ex ante decision, the policy-maker sacrifices information if he sets a prohibitive transfer. By Proposition 1, he takes such action only if access is ex ante and $a_{\varnothing}=1$. Consequently, $a_{0}=a_{1}=1$. Hence, we expect an extra 
status-quo bias toward the high action for any signal realization, if the transfer is prohibitive.

The two biases move in the opposite directions. Hence, it is interesting to understand which bias dominates if both biases are present at the same time. The next proposition derives the key observation; the status-quo bias exactly corrects for the negative externality. Somewhat surprisingly, the information loss is associated with a welfare-improving action, not vice versa.

Proposition 2 (Prohibitive transfer, non-benevolent policy-maker) If the transfer is prohibitive and the policy-maker is non-benevolent, then welfare is maximized.

Proof. Consider $s=1$. By Proposition 1, a necessary condition for a prohibitive transfer is $a_{\varnothing}=1$, which implies that $\pi_{\varnothing}>\frac{1}{2}$ and $a_{0}=a_{1}=1$. Then, $\pi_{1} \geq \pi_{\varnothing}>\frac{1}{2}>\frac{V-1}{2 V}$, and consequently, $a_{1}^{*}=a_{1}$. There is no welfare distortion for the high signal realization, $s=1$.

Consider the low signal realization, $s=0$. If $a_{0}^{*}=1$, then $a_{0}^{*}=a_{0}$, and there is no welfare distortion. If $a_{0}^{*}=0$, then $\pi_{0}<\frac{V-1}{2 V}$, or equivalently,

$$
\beta<1-\alpha \frac{\pi_{\varnothing}}{1-\pi_{\varnothing}} \frac{V+1}{V-1} .
$$

However, eq. [11] is inconsistent with the negative surplus, which is a necessary condition for access restriction. By the zero-surplus equation in eq. [8], the negative surplus requires

$$
\beta>1-\alpha \frac{\pi_{\varnothing}}{1-\pi_{\varnothing}} \frac{V+1}{V-1}
$$

The policy-maker's prohibitive transfer increases the welfare because the two distortions cancel each other out. Put more precisely, the status-quo bias serves as a check for the externality bias; it guarantees the first-best action independently of the size of the externality bias. Thereby, the policy-maker ex ante improves the welfare relative to her ex post (non-contractible) actions.

Finally, we can briefly discuss the equilibrium properties when the lobby participates. By Proposition 2, we know that the only distortion occurs toward the low action. Hence, in terms of welfare, there are only three possibilities:

- No distortion: If $\left(a_{0}, a_{1}\right)=(1,1)$, then $\left(a_{0}^{*}, a_{1}^{*}\right)=(1,1)$. This is one of the cases in which the equilibrium entails the first-best allocation. This 
equilibrium occurs if the status-quo action is the high action, and the signal is uninformative. ${ }^{5}$

- Single distortion: For a single distortion, we must have the separating equilibrium, $\left(a_{0}, a_{1}\right)=(0,1)$, and the first-best actions, $\left(a_{0}^{*}, a_{1}^{*}\right)=(1,1)$. An example is the case of $\pi_{1}>\frac{1}{2}>\pi_{\varnothing}>\pi_{0}>\frac{V-1}{2 V}$. These parameters guarantee that the lobby participates and that the signal is informative.

- Double distortion: The existence of double distortion can be easily evaluated. Double distortion occurs if and only if $\left(a_{0}, a_{1}\right)=(0,0)$ and $\left(a_{0}^{*}, a_{1}^{*}\right)=(1,1)$. This equilibrium occurs only if the signal is uninformative and $a_{\emptyset}=0$ (or, equivalently $\pi_{\varnothing} \leq \frac{1}{2}$ ). To obtain high actions that are socially optimal, we must have $\pi_{0}>\frac{V-1}{2 V}$. Because the signal is uninformative, we must also have $\pi_{1} \leq \frac{1}{2}$. To sum up, a necessary condition for double distortion is $\frac{1}{2} \geq \pi_{1} \geq \pi_{0}>\frac{V-1}{2 V}$.

\subsection{Benevolent policy-maker with a mixed objective}

Suppose the welfare measure is the sum of all players' expected losses from the policy-maker's actions. As in Grossman and Helpman (2001), the policy-maker's objective is mixed. On one hand, the policy-maker is benevolent and accounts for the total expected losses (i.e. welfare). On the other hand, the policy-maker values her private benefits.

To obtain explicit microfoundations for the welfare, assume that we have three agents. Only Agent 1 is organized and can meet the policy-maker; hence, Agent 1 becomes a single lobby. (i) Agent 1's loss function is $l_{1}(a)=1-a$. (ii) Agent 2's loss function is $l_{2}(a)=a$. (iii) Agent 3's loss function is $l_{3}(a, \theta)=L(a, \theta)$. The policy-maker has an action-independent loss function; alternatively, we may think of him as being either Agent 2 or Agent 3.

By definition, the total welfare loss is $W(a, \theta)=l_{1}(a)+l_{2}(a)+l_{3}(a)-1=$ $L(a, \theta)$. Hence, ex post, the benevolent policy-maker with a mixed objective and posterior $\pi$ sets the welfare-maximizing action $a \in\{0,1\}$ exactly as in the previous analysis, namely to minimize $E(a, \pi)$.

Ex ante, however, private gains matter. In the policy-maker's mixed objective, the parameter of benevolence is $\lambda$. Hence, the policy-maker's expected loss in signal realization $s$, is

$$
U(a, s):=\lambda\left[\left(1-\pi_{s}\right) W(a, 0)+\pi_{s} W(a, 1)\right]-(1-\lambda) e_{s} c .
$$

5 Notice that this case does not exhaust the full set of non-distorting equilibria. 
When setting the access fees, the policy-maker's objective is to minimize the ex ante loss, $(1-f) U\left(a_{0}, 0\right)+f U\left(a_{1}, 1\right)$.

Finally, to keep our setting intact, the values of the fees must be identical for the lobby (Agent 1) and the policy-maker. The policy-maker treats her private gains exactly as the welfare loss of lobby only if $\lambda=\frac{1}{2}$. Obviously, this assumption does not imply that the policy-maker is a genuine altruist who treats the private benefits of the other agents as being equal to her private gains. In fact, the policy-maker discriminates between the sources of the losses of the other agents: (i) The loss of any agent is fully accounted for only if it is associated with the action that disfavors the agent. (ii) In contrast, the loss of an agent stemming from a private transfer to policy-maker is not considered at all. This dichotomy is important for the construction and interpretation of the welfare properties of the equilibrium.

Clearly, in this setting, the participation of the lobby is equivalent to having the maximal welfare. The welfare distortion arises only if the information available to the benevolent policy-maker in the final Stage 4 is incomplete. Proposition 3 builds a result that is parallel to Proposition 2.

Proposition 3 (Prohibitive transfer, benevolent policy-maker) If the transfer is prohibitive and the policy-maker is benevolent, then welfare is not maximized.

Proof. By Proposition 1, the signal is informative if transfer is prohibitive; hence, $\left(a_{0}^{*}, a_{1}^{*}\right)=(0,1)$. However, given non-participation, we have $\left(a_{0}, a_{1}\right)=(1,1)$. Hence, there is a single distortion at low signal realization, $a_{0} \neq a_{0}^{*}$.

The interpretation of the welfare loss in this setting is straightforward. The policy-maker faces a tradeoff between the amount of information (public benefits) and the amount of money (private benefits). Since the two types of benefits are weighted one to one and the policy-maker has full bargaining power, the typical scenario is as follows: the policy-maker encourages the disclosure of the privately informed lobby, which maximizes the public benefits. To do so, the policy-maker compensates the lobby for the potential adverse effect of disclosure on the privately informed lobby (i.e. offers a compensation) or exploits the potential beneficial effect of disclosure on the lobby (i.e. requires a fee). The proposed transfer exactly restores the lobby's outside option. Thus, the policy-maker can both maximize the public benefits and extract part of the public benefits to himself.

However, this scenario does not work if the lobby's outside option is high and the extra public benefits are low. In such a setting, the compensation is prohibitively costly, and the policy-maker sacrifices increasing the public 
benefits for savings private costs. Unlike the previous setting with a non-benevolent policy-maker, this setting with a mixed objective yields a genuine tradeoff between the amounts of private and public benefits.

\section{Extensions}

\subsection{Preferences regarding information structures}

We first derive the partner's preferences regarding noise $(\alpha, \beta)$. The preferences depend primarily on the status-quo action:

- High (favorable) status-quo action, $a_{\varnothing}=1$ : Clearly, the partner prefers an uninformative signal, which implies that the surplus is zero and that the partner defends the high action.

- Low (unfavorable) status-quo action, $a_{\varnothing}=0$ : For both timings, the partner participates. Hence, the partner always obtains the payoff of her outside option, which is the value of the low action. Thus, the partner is indifferent regarding noise.

The policy-maker's preferences are given by the maximization of the level of surplus. ${ }^{6}$

- High (favorable) status-quo action, $a_{\varnothing}=1$, and ex ante access: In Section 3.5, we observed that the shape of the iso-surplus curves depends on the relative valuation: (i) If $V \geq 1$, the surplus is maximized at noiseless signal $(\alpha, \beta)=(0,0)$. (ii) If $V<1$, then the surplus is maximized at an uninformative signal.

- Low (unfavorable) status-quo action, $a_{\varnothing}=0$, or interim access: By inspection of eqs [2] and [3], the iso-surplus curves have the same slope as that identified in Section 3.5. The only difference is that the surplus level is always non-negative because the surplus is monotonic in noise for the informativeness condition. Thus, the maximal surplus depends on the relative valuation: (i) If $V \geq 1$, the surplus is maximized at noiseless signal $(\alpha, \beta)=(0,0)$. (ii) If $V<1$, then the surplus is maximized in the neighborhood of the biased informative signal $(\alpha, \beta) \rightarrow(0,1)$. In this interesting

6 Notice that the surpluses $S$ and $H$ are identical under $a_{\phi}=0$ or an uninformative signal and differ only under $a_{\varnothing}=1$ and an informative signal. 
scenario, any incremental transfer from the lobby compensates an incremental policy loss, hence the policy-maker maximizes extraction by maximizing the frequency of the high types.

To sum up, in a standard scenario $(V \geq 1)$, the objectives of the partner and the policy-maker are exactly opposite. In an alternative scenario $\left(V<1\right.$ and $\left.a_{\varnothing}=1\right)$, both players prefer an uninformative signal and there is no conflict between the policy-maker and the lobby over the information structure.

\subsection{Non-skeptic policy-maker}

Relaxing the assumption of full disclosure (Assumption 1) has no effect on the equilibrium payoffs as long as we maintain weak dominance.

- Consider the high type of the lobby. Non-disclosure $\left(d_{1}=0\right)$ is weakly dominated by disclosure $\left(d_{1}=1\right)$. The posterior belief under disclosure is $\pi_{1}$, while the posterior belief under non-disclosure for any mixedstrategy profile lies in the interval $\left[\pi_{0}, \pi_{1}\right]$. The lobby's payoff is increasing step wise in the posterior; hence, the action that maximizes the posterior weakly dominates all other actions.

- Consider the low type of the lobby. The argument above shows that the high type of the lobby discloses evidence $\left(d_{1}=1\right)$ under weak dominance. Thus, the posterior under non-disclosure, for any profile including a mixedstrategy profile, is equal to $\pi_{0}$. The posterior under disclosure is also $\pi_{0}$. Thus, the policy-maker's action $a$ remains constant. The low type of the lobby is indifferent regarding any combination of disclosure and nondisclosure.

In contrast, allowing a pooling equilibrium under interim access (i.e. relaxing Assumption 2) may have an effect. The pooling profile is $\left(e_{1}, e_{2}\right)=(0,0)$. The posterior belief for non-participation is now $\pi_{\phi}$, which implies status-quo action $a_{\phi}$. Thus, the outside option (participation condition) of the high type of the lobby is now characterized by the status-quo action, not the low action. As a result, the bargaining surplus for interim access under a pooling profile is

$H_{p}\left(\alpha, \beta, \pi_{\varnothing}\right):=\left\{\begin{array}{cc}I\left(\alpha, \beta, \pi_{\phi}\right)\left[E_{\varnothing}\left(\pi_{\varnothing}\right)-E_{e}\left(\alpha, \beta, \pi_{\varnothing}\right)+f\left(\alpha, \beta, \pi_{\varnothing}\right)-1\right] \gtreqless 0 & \text { if } \pi_{\varnothing}>\frac{1}{2}, \\ I^{\prime}\left(\alpha, \beta, \pi_{\varnothing}\right)\left[E_{\varnothing}\left(\pi_{\varnothing}\right)-E_{e}\left(\alpha, \beta, \pi_{\varnothing}\right)+f\left(\alpha, \beta, \pi_{\varnothing}\right)\right] \geq 0 & \text { if } \pi_{\varnothing} \leq \frac{1}{2} .\end{array}\right.$ 
By comparing eqs [14] and [2], we find that $H_{p}\left(\alpha, \beta, \pi_{\varnothing}\right)=S\left(\alpha, \beta, \pi_{\varnothing}\right)$. Since a pooling profile is an equilibrium only if the high type of the lobby does indeed not participate, $H_{p}\left(\alpha, \beta, \pi_{\varnothing}\right)<0$, it exist under interim access if and only if the transfer is prohibitive also under ex ante access. In a word, the existence of a pooling equilibrium eliminates any difference between the interim and ex ante access.

\section{Conclusions}

This paper develops a disclosure game with a single lobby and a single policymaker in which the policy-maker announces a transfer that conditions the disclosure of evidence of the lobby. The game is close to the family of access fee models that originated with Austen-Smith (1995, 1998), Ball (1995), and Lohmann (1995) and that were recently extended by Cotton (2009, 2012). The main difference is that the model allows for both positive transfers (access fees) and negative transfers (access compensations), which requires the commitment of both players, whereas the access fee models involve only a single commitment.

A conceptual contribution of the model is to demonstrate that the existence of two commitments conditional on the realization of the transfer is equivalent to the existence of a bilateral transaction. The transaction is modeled as a simple non-cooperative bargaining game. The policy-maker makes a single take-it-or-leave-it offer, and her bargaining partner agrees or disagrees with the offer. The bargaining partner is either a lobby (if meeting precedes the private signal) or a high type of the lobby (if meeting follows the private signal). The main lesson is that the lack of communication is equivalent to the transaction not being realized, which is equivalent to the negative sign of the bargaining surplus. Therefore, for any timing, noise, valuation, and beliefs, it is sufficient to calculate the sign of the surplus to obtain the equilibrium.

Our main focus has been on the conditions under which the surplus is negative. In such a case, the policy-maker announces a prohibitive transfer, and the verifiable evidence is not presented to the policy-maker. We identify the negative surplus (and non-participation of the lobby) only if the lobby has an attractive outside option. This explanation of the absence of communication is very different from search models of lobbying. In these models, the barrier to the transmission of verifiable information is that low types do not participate with the aim of pooling unfavorable evidence with a lack of evidence (Bennedsen and Feldmann 2006; Dahm and Porteiro 2008; Henry 2009). 
Another main lesson of the paper is that the normative assessment of the prohibitive transfer and the ensuing information loss crucially depends on the policy-maker's objective. If the policy-maker is non-benevolent, then the lack of communication restriction always improves the overall welfare, and the policy-maker's uninformed actions are the first-best actions. In contrast, if the policy-maker is benevolent, then the lack of communication restriction always deteriorates the overall welfare, and the policy-maker's uninformed actions are not the first-best actions.

Additionally, we analyze the preferences regarding information structures (signal noise). In the absence of transfers, the policy-maker always prefers a noiseless signal, and the lobby prefers a signal that maximizes the probability of the favorable action. With transfers, there are various scenarios in which the preferences can be perfectly aligned but also perfectly opposite between the lobby and the policy-maker. Whenever the policy-maker's valuation of the policy exceeds the lobby's valuation, we obtain the strongest conflict: the policy-maker prefers noiseless evidence, whereas the lobby prefers uninformative evidence. In contrast, if the lobby's valuation of the policy exceeds the policy-maker's valuation and the status-quo policy is favorable for the lobby, then both players prefer uninformative evidence. If the lobby's valuation of the policy exceeds the policy-maker's valuation, there is also a scenario in which the policy-maker prefers a distorted but informative signal that maximizes the probability of the favorable action.

Acknowledgments: I would like to thank three anonymous reviewers, Jan Zápal, and participants at the EPCS 2013 conference in Zürich and the CEPET 2014 workshop in Udine for their useful comments. Financial support from the Grant Agency of the Czech Republic No. P402/12/G097 is gratefully acknowledged.

Funding: Grant Agency of the Czech Republic P402/12/G097.

\section{References}

Ansolabehere, S., J. M. Snyder, and M. Tripathi. 2002. "Are PAC Contributions and Lobbying Linked? New Evidence from the 1995 Lobby Disclosure Act." Business and Politics 4(2):131-55.

Austen-Smith, D. 1995. "Campaign Contributions and Access." American Political Science Review 89:566-81.

Austen-Smith, D. 1998. "Allocating Access for Information and Contributions." Journal of Law, Economics, and Organization 14(2):277-303. 
Ball, R. 1995. “Interest Groups, Influence and Welfare." Economics \& Politics 7(2):119-46. Baron, D. 1989. "Service-Induced Campaign Contributions and the Electoral Equilibrium." Quarterly Journal of Economics 104:45-72.

Bennedsen, M., and S. E. Feldmann. 2006. "Informational Lobbying and Political Contributions." Journal of Public Economics 90:631-56.

Bertrand, M., M. Bombardini, and F. Trebbi. 2014. "Is It Whom You Know or What You Know? An Empirical Assessment of the Lobbying Process.” American Economic Review 104(12): 3885-920.

Bhattacharya, S., and A. Mukherjee. 2013. "Strategic Information Revelation When Experts Compete for Influence." RAND Journal of Economics 44(3):522-44.

Cho, I., and D. M. Kreps. 1987. "Signaling Games and Stable Equilibria." Quarterly Journal of Economics 102(2):179-221.

Cotton, C. 2009. "Should We Tax or Cap Political Contributions? a Lobbying Model with Policy Favors and Access." Journal of Public Economics 93:831-42.

Cotton, C. 2012. "Pay-to-Play Politics: Informational Lobbying and Contribution Limits When Money Buys Access." Journal of Public Economics 96:369-86.

Dahm, M., and N. Porteiro. 2008. "Side-Effects of Campaign Finance Reform." Journal of the European Economic Association 6(5):1057-77.

Dewatripont, M., and J. Tirole. 2005. "Modes of Communication." Journal of Political Economy 113(6):1217-38.

Dziuda, W. 2011. "Strategic Argumentation.” Journal of Economic Theory 146:1362-97.

Esterling, K. M. 2007. "Buying Expertise: Campaign Contributions and Attention to Policy Analysis in Congressional Committees.” American Political Science Review 101(1):93-109.

Grossman, G. M., and E. Helpman. 2001. Special Interest Politics. Cambridge, MA: MIT Press.

Henry, E. 2009. "Strategic Disclosure of Research Results: The Cost of Proving Your Honesty." Economic Journal 119:1036-64.

Kerr, W. R., W. F. Lincoln, and P. Mishra. 2011. “The Dynamics of Firm Lobbying.” NBER Working Paper 17577.

Lohmann, S. 1995. "Information, Access, and Contributions: A Signaling Model of Lobbying." Public Choice 85:267-84.

Milgrom, P. 1981. "Good News and Bad News: Representation Theorems and Applications." Bell Journal of Economics 12:350-91.

Milgrom, P., and J. Roberts. 1986. "Relying on the Information of Interested Parties." RAND Journal of Economics 17:18-32.

Richter, B. K., K. Samphantharak, and J. F. Timmons. 2009. "Lobbying and Taxes." American Journal of Political Science 53(4):893-909.

Seidmann, D. J., and E. Winter. 1997. "Strategic Information Transmission with Verifiable Messages." Econometrica 65:163-9.

Shin, H. 1994. "The Burden of Proof in a Game of Persuasion." Journal of Economic Theory 64:253-64.

Snyder, J. M. 1990. "Campaign Contributions as Investments: The U.S. House of Representatives 1980-1986.” Journal of Political Economy 98:1195-227.

Wright, J. R. 1996. Interest Groups and Congress: Lobbying, Contributions, and Influence. Boston, MA: Allyn \& Bacon. 